\title{
ADAPTIVE MULTISTAGE VECTOR QUANTIZATION
}

\author{
José A. Rodríguez Fonollosa and Enrique Masgrau
}

D. Teoria del Senyal i Comunicacions. UPC. Spain

\begin{abstract}
SUMMARY
In this paper, we present a new multiple stage vector quantization method that allows the adaptation of the quantizer to signal to be coded. This adaptation is computationally very simple and is made with no increase in bit-rate. The resulting quantizer provides a robust performance across different speakers and environments. It has been applied to the quantization of the LPC parameters and the results show a significant increase in performance respect to conventional multi-stage vector quantizers.
\end{abstract}

\section{INTRODUCTION}

Most of the low-bit-rate coders that have been developed recently include one or various vector quantizers in their scheme. Vector quantization (VQ) is a process of redundancy removal that makes effective use of four interrelated properties of vector parameters: linear dependency (correlation), nonlinear dependency, shape of probability density function (pdf), and vector dimensionality itself [1].

Previous work on VQ usually employed trained codebooks or codebooks populated with randomly generated samples. In the first case, it is important to use long and representative training sequences to obtain a good performance "out" of the training sequence. Even though, the performance of any codebook is expected to deteriorate if used with different speakers and environments. In the second case, the quantizer is not taking advantage of the four interrelated properties and the performance reduces.

It is well known that, for a given bit rate, a speaker-dependent codebook, i.e., designed for the specific speaker whose speech is being coded, would work better than a speakerindependent codebook. This fact has motivated our research of a VQ system that adapts its codebook as it is used. This system would have the extra advantage of also automatically adapting to the acoustic environment of the speaker and to the recording conditions.

This idea is not completely new and, for example, in [2] a system that changes the code-vectors in time is described. Nevertheless this change creates the necessity of transmitting the now vectors to the receiver with a significant increase in bit-rate. In this paper, we describe a simple algorithm for updating the codebook that eliminates the necessity of very long and representative training sequences, and allow the quantizer to be continuously adapted to the signal statistics with no increase in bit-rate. This adaptation provides a better signal to noise ratio and robust performance across differents speakers and speech recording conditions.

In the last part of the paper, it is shown that the application of this Adaptive Multi-Stage Vector Quantization (AMSVQ) technique to the quantization of the log-area ratios (LAR) give very good results. Accurate quantization of LPC parameters is necessary for obtaining high quality speech at low bit-rates and AMSVQ is shown to be a good candidate to quantize the short-term predictor coefficients in multipulse excitation coders and codebook excited coders.

\section{DESCRIPTION}

Multi-stage has always been seen as a suboptimal VQ scheme with reduced complexity and storage. It consists of successively approximating the input vector in several cascaded VQ stages, where the input vector from each stage is the quantization error from the preceding stage. In [3] the multiple stage vector quantization is applied to the quantization of the LPC parameters, and it is shown that, in the case of Euclidean distance measures such as the log- 
area ratio measure, that quantizer is very close to a theoretically predicted asymptotically optimal rate distortion relationship.

In the scheme we present the multi-stage structure has been used to develop a continuously adaptive VQ system. The main idea of the adaptation algorithm is to update the preceding codebooks taking into account the information given by the rest of the quantizers. In the most simple scheme, with 2 stages, a vector $x$ is quantized to give the quantized vector $z$

$$
z=c+e_{q}
$$

where $\mathrm{c}$ is the output of the first quantizer and eq the contribution of the second codebook. (See figure 1).

$$
\begin{aligned}
& c=q 1(x) \\
& e_{q}=q 2(x-c)
\end{aligned}
$$

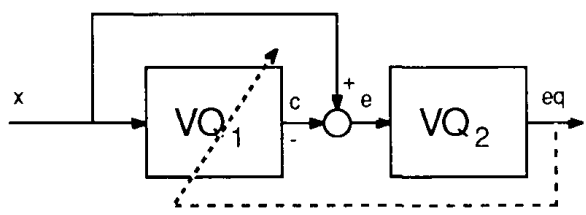

Figure 1. AMSVQ system with 2 stages

Then eq, that is an estimation of the quantization error of the first codebook, can be used to adapt the quantizer in the following way:

$$
c=c+\mu e_{q}
$$

where $\mu$ is the adaptation factor. This adaptation is made without the need of increasing bit-rate and is computationally very simple. It can be seen as a modification of the centroid in the direction of the new quantized vector and, in this sense, its closely related with the LBG algorithm [6]. The difference between both algorithms is that the AMSVQ system is continuously changing the centroids and uses an estimation of the error (or input vector), while the LBG algorithm changes the centroid after classifying all the training sequence and uses the actual input vector during this training phase.

This algorithm can also be obtained applying a LMS-type minimization algorithm to the error at the output of the first codebook. The LMS algorithm is widely used in adaptive systems and it is a steepest descent algorithm where the gradient of the averaged distortion is replaced by the gradient of the current distortion $d$. The general equation of this algorithm is:

$$
w=w-\frac{1}{2} \mu \nabla_{w} d
$$

If we consider a squared error distortion measure

$$
D=E\{d\}
$$

where the instantaneous distortion $d$ is

$$
d=e^{2}=(x-c)^{2}
$$

and its gradient

$$
\nabla_{c} d=-2 e
$$

we obtain the adaptation equation

$$
c=c+\mu e
$$

as $\mathbf{e}$ is not available at the receiver, the algorithm uses the estimation $\mathrm{e}_{\mathrm{q}}$ given by the rest of the quantizers and we obtain equation (4).

In fact, similar algorithms has been described in the literature to design the codebook but is the first time, as we know, that it is used to redesign the codebook as the vector quantizer is used. In [7] gradient algorithms are used for designing Predictive Vector Quantizers and the stochastic gradient formula (9) was derived for updating the quantizer. In [5] Kohonen presents an algorithm for producing "self organizing feature maps", i.e., vector quantizers, that uses the actual error e to update, not only the codeword $c$ but also all the codewords in the "neighbourhood". Nevertheless this extension of the adaptation algorithm to the neighbourhood represents an important increase in the complexity of the system and the simulations show no improvement in the performance of the AMSVQ system.

The codebooks can be full searched codebooks or gain-shape codebooks with randomly generated values. We have investigated the performance of the AMSVQ system with full searched codebooks and the LAR parameters but gain-shape codebooks and gain adaptive quantizers [4] can be also a good choice for this and other applications.

Although robust to variations in the signal statistics the above adaptation make the quantizer more sensitive to channel errors that the conventional multi-stage structure. This problem can be reduced using a leakage factor similar to that used in the LMS-type algorithms of practical ADPCM schemes. Then (4) is modified to give:

$$
C=c(1-B)+\mu e q+B c_{0}
$$

where $B$ is a small value that control the memory of the system and $c_{0}$ is the initial value of the codeword $c$. 


\section{DESIGN OF THE CODEBOOKS}

The codebook design approach is similar to that used in conventional multi-stage VQ [1]. We need a representative speech training set from different speakers, but thanks to the adaptive nature of the AMSVQ system the results are not expected to be very conditioned by the election of the training sequence.

Starting with the first codebook and using the LBG clustering algorithm [6], the codebooks are constructed in succession. The training sequence for the second codebook is obtained as the first codebook quantization error. The problem is that to obtain this quantization error, we need the output of the second codebook to apply the adaptation algorithm (5) to the first codebook. As e $q$ is not available to obtain this training sequence, the actual quantization error $e$ is used to adapt the first codebook.

After obtaining, by this method, a first version of the codebooks the process can be repeated to improve the design. Now the output of this previous quantizers can be used to update the first quantizer and a more representative training sequence for the rest of the quantizers is obtained. This close-loop design was found to provide about $1 \mathrm{~dB}$ improvement over the first open-loop design for the training sequence. Nevertheless, the results for the test sequence are similar in both cases.

\section{BESULTS}

The AMSVQ system has been applied to the quantization of LPC parameters with important improvements respect to the conventional multistage structure. Quantization of the LPC coefficients has been extensively studied. Generally, the inverse sines of the reflection coefficients or the log-area ratio values are quantized. We chose the log-area ratio values with the mse distortion for our experiments.

Vector quantizers have proven to be very efficient in encoding the predictor parameters. Nevertheless for high quality coding $24-30$ bits are required and a full search codebook become impractical. Structured codebooks as the multistage must be used to reduce the complexity. The developed adaptation algorithm makes the suboptimal multi-stage structure efficient and robust against different speakers, languages and environments.

To design the quantizer a training speech sequence formed by 42 sentences of 3 female and 3 male speakers was used. And to test the quantizers we chose 24 sentences of a different male speaker (not included in the training sequence). The silent segments (background noise) were not processed.
The speech signal was not preemphasized and the correlation method with a Hamming window of 200 samples (25 ms) was used to obtain ten log area parameters every 160 samples (20 ms).

The results have been obtained using a codebook of 8 codewords ( 3 bits/frame) in the first stage and 3 codebooks of 256 codewords ( 8 bits/frame) in the second, third and fourth stage. The number of bits for each frame is thus $27(3+8+8+8)$ and at $50 \mathrm{frames} / \mathrm{s}$, the bit rate equals $1350 \mathrm{bps}$. To obtain a fast adaptation and robustness against channel errors, only the 8 codewords of the first quantizer are adapted.

First of all, we studied the performance of a single codebook of 8 codewords ( 3 bits) when it was adapted using the actual error e (forward adaptation). The results (figure 2) show an increase in signal to noise ratio (SNR) of more of $2 \mathrm{~dB}$ for values of the step size between 0.1 and 1. Two local maximum can be observed near $\mu=$ 0.1 and $\mu=0.6$, so these values and $\mu=0$ were used to design three different sets of codebooks.

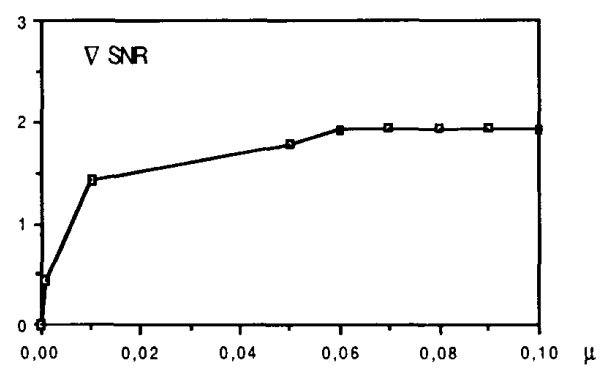

Figure 2a. Increase in SNR for a single codebook with forward adaptation and step sizes between 0 and 0.1 .

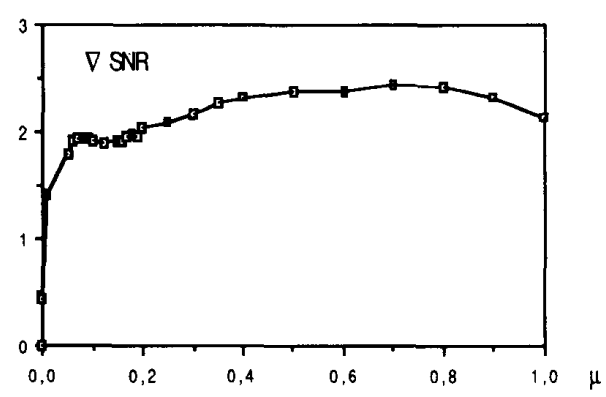

Figure 2b. Increase in SNR for a single codebook with forward adaptation and step sizes between 0 and 1 . 
Then, the designed codebooks have been used to quantize the test sequence with different values of the step size $\mu$. In figure 3 it is shown the increment in SNR respect to $19 \mathrm{~dB}$ of the conventional, $(\mu=0)$, multistage vector quantizer. The best results are obtained with $\mu=0.4$ and the AMSVQ system designed for $\mu=0.1$. Nevertheless smaller values of the step size as $\mu=0.1$ or $\mu=0.01$ give also a significant increase of the performance and can be a good chocie for providing robustness against channel errors.

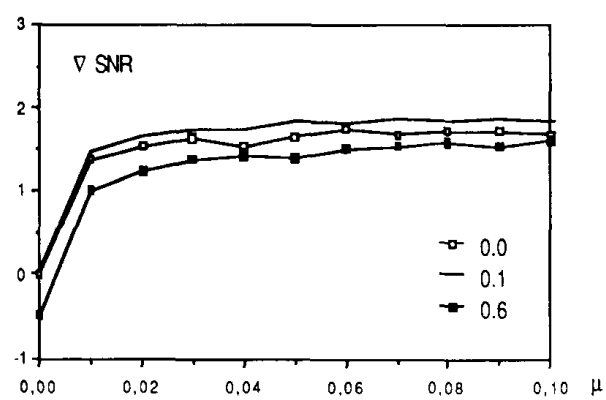

Figure 3a. Increase in SNR for the three designed AMSVQ and step sizes between 0.0 and 0.1

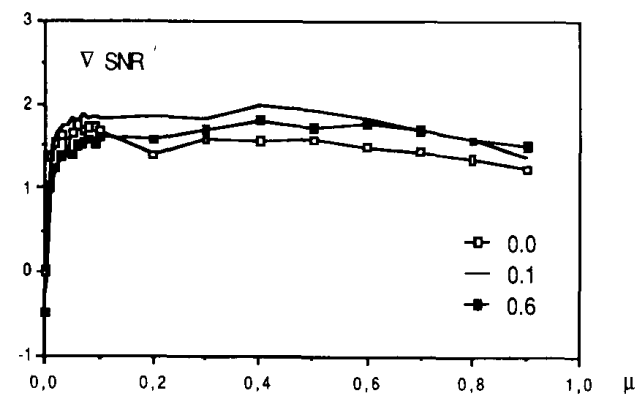

Figure 3b. Increase in SNR for the three different designed $A M S V Q$ and step sizes between 0.0 and 1 .

This quantization of the LPC parameters has been included in a CELP coder. The results show that the coded speech can hardly be distinguish from the one obtained with unquantized coefficients. The signal to noise ratio of the coded speech is also similar in both cases.

\section{CONCLUSIONS}

The application of the developed adaptation algorithm to the multiple stage vector quantizer makes it robust across different speakers, languages and environments. This algorithm is very simple and requires no additional bits. The quantizer is continuously redesigned and it is less sensible to the chosen training sequence.

The results show that the AMSVQ increases the performance of conventional multistage quantizers and can be a good choice for coding the LPC coefficients with high quality.

Only full searched codebooks have been considered here. Gain-shape or gain adaptive vector quantizers can also be a good choice and we are currently working in the application of the AMSVQ system with these variations to other coding schemes.

Future work includes also an exhaustive study of the influence of the number of adaptive codewords and stages in the performance of the quantizers.

\section{BEERBNCES}

[1] J. Makhoul, S. Roucos and H. Gish. "Vector Quantization in Speech Coding". Proc. IEEE, vol. 73, NOV-1985.

[2] D. B. Paul. "An 800 bps adaptive vector quantization vocoder using a perceptual distance measure". IEEE Proc. ICASSP, Boston, APR-1983.

[3] B-H. Juang and A. H. Gray, Jr. "Multiple Stage Vector Quantization for speech coding". IEEE Proc. ICASSP, Paris, MAY-1982.

[4] J-H. Chen and A. Gersho. "Gain-Adaptive Vector Quantization with Application to Speech Coding". IEEE Trans. Communications. SEP-1987.

[5] T. Kohonen.Self-Organization and Associative Memory. Springer-Verlag, Berlin 1988

[6] Y. Linde, A. Buzo, R. M. Buzo and R. M. Gray. "An Algorithm for Vector Quantizer Design". IEEE Trans. on ASSP, No. 4, AUG-1980.

[7] Pao-Chi Chang and Robert M. Gray. "Gradrient Algorithms for Designing Predictive Vector Quantizers". IEEE Trans. on ASSP. Vol. 34, No. 4, AUG-1986. 